\title{
Facts, scope, style: a guide to writing papers for IEEE transactions on consumer electronics
}

Article

Accepted Version

Sherratt, S. (2014) Facts, scope, style: a guide to writing papers for IEEE transactions on consumer electronics. IEEE Consumer Electronics Magazine, 3 (3). pp. 49-52. ISSN 21622248 doi: https://doi.org/10.1109/MCE.2014.2317889 Available at https://centaur.reading.ac.uk/39290/

It is advisable to refer to the publisher's version if you intend to cite from the work. See Guidance on citing.

To link to this article DOI: http://dx.doi.org/10.1109/MCE.2014.2317889

Publisher: IEEE

All outputs in CentAUR are protected by Intellectual Property Rights law, including copyright law. Copyright and IPR is retained by the creators or other copyright holders. Terms and conditions for use of this material are defined in the End User Agreement.

www.reading.ac.uk/centaur

\section{CentAUR}


Central Archive at the University of Reading

Reading's research outputs online 
Full text version

A guide to writing papers for

IEEE Transactions on Consumer Electronics.

\section{R. Simon Sherratt}

IEEE Consumer Electronics Magazine, volume 3, issue 3, 3 July 2014

Digital Object Identifier 10.1109/MCE.2014.2317889

http://dx.doi.org/10.1109/MCE.2014.2317889

In a previous article, I wrote a brief piece on how to enhance papers that have been published at one of the IEEE Consumer Electronics (CE) Society conferences to create papers that can be considered for publishing in IEEE Transactions on Consumer Electronics (T-CE) [1]. Basically, it included some hints and tips to enhance a conference paper into what is required for a full archival journal paper and not fall foul of self-plagiarism. This article focuses on writing original papers specifically for T-CE.

After three years as the journal's editor-in-chief (EiC), a previous eight years on the editorial board, and having reviewed some 4,000 T-CE papers, I decided to write this article to archive and detail for prospective authors what I have learned over this time. Of course, there are numerous articles on writing good papers-some are really useful [2], but they do not address the specific issues of writing for a journal whose topic (scope) is not widely understood or, indeed, is often misunderstood.

\section{T-CE FACTS}

T-CE is now in its 60th year. It currently receives some 1,200 submitted papers per year, and the number of submissions is increasing. There are four issues per year, electronic and print. Each issue has a submission deadline, and when papers are submitted before the relevant deadline, the paper will normally be considered for the following issue. T-CE currently has the fastest average turnaround between submission to e-publication for IEEE journals-but this does come with strict unmovable deadlines. T-CE only employs minimal editorial staff, so authors need to submit publication-ready papers for consideration that are exact to the template. 
T-CE is the primary journal to enable the publication of new, topical, and interesting research in the field of consumer electronics. T-CE papers must present new fundamental science, but the papers must also be in scope, enhance the field of $\mathrm{CE}$, and meet the basic guidelines.

\section{SCOPE}

Authors often get confused about the scope of T-CE or make incorrect assumptions and submit totally inappropriate papers. While there are extreme cases of inappropriate technologies for CE that have been submitted for consideration (underwater cables, satellite batteries, brain implants, etc.), even more mainstream technologies still need to be specifically placed in the CE scope; otherwise, it is not a CE paper. Currently, the percentage of papers accepted is around $10 \%$; hence, nine out of ten papers are rejected, and, of those nine rejects, approximately four to six papers are rejected simply because the paper is not in the scope of T-CE or the authors have not read the instructions.

I often get asked by research students how long the review process is because they want to publish as early as possible. While this enthusiasm is admirable, if the paper is out of scope, then they are wasting their time. Sometimes the scope of a journal is rather obvious, for example, IEEE Transactions on Antennas and Propagation (T-AP), IEEE Transactions on Education, or IEEE Transactions on Industrial Electronics. However, when it comes to T-CE, authors often assume that just because CE devices contain many technologies, their paper must be in the scope of T-CE-after all, many CE devices contain antennas and power supplies. However, by implication, it is not always true that the paper is within the scope of CE, and whether a paper is in scope depends on its focus. The actual scope of T-CE is aligned with the field of interest of the CE Society and is defined as:

The engineering and research aspects of the theory, design, construction, manufacture, or end use of mass market electronics, systems, software, and services for consumers.

While the T-CE scope is rather wide, the scope is deliberately and clearly focused on mass-market systems for consumers. Papers that discuss one-off developments that will have very limited market appeal or papers that do not consider the consumer aspect are simply out of scope. Furthermore, IEEE journals do not accept papers that are obviously in the scope of other IEEE journals. Therefore, to take the example above, $\mathrm{CE}$ devices do often contain antennas and benefit from antenna developments, but these developments should be published in T-AP, not T-CE, likewise for other technologies. Indeed, the question arises as to why authors would want to publish a paper on antenna design in T-CE and not TAP, likewise for other topics including multimedia, broadcasting, power electronics, etc. Because the field of CE embraces a host of different technologies, I do feel that some authors try to use T-CE as a back door to publishing papers on specific technologies rather than submitting their paper to a more topic-specific journal. The editorial board is aware of these practices, and such a back door does not exist.

Perhaps another example closer to home illustrates the point. T-CE publishes papers on video codecs and image processing, but other journals do too, so what is the difference? For T-CE, authors need to 
show new developments (ideas, algorithms, systems, or services) as is required for any archival paper and also demonstrate that their work is indeed of benefit to the CE community, perhaps by enhancing usage, deployment, new features, lower power, less memory, usability, etc. In many cases, a new video codec or image processing algorithm that has been simulated on a PC simply does not show it is of benefit to CE. Could the algorithm run in real time on a typical constrained embedded CE device-and has this been proven? What CE standard, product, or service would benefit from using this technology? Have the authors considered how this technology can be deployed into CE devices? Without these dimensions to the paper, the paper is not a CE paper and, in all likelihood, should be published elsewhere. Therefore, authors need to demonstrate that their work is scientifically sound and suitable for the journal. For T-CE, authors do not need to have made a prototype device, but in some cases, this can be useful. However, they do need to have demonstrated the work can be of real benefit for current or future CE devices. Evidence can come in many forms, but often comes in complexity analysis, comparisons with previous CE research, field trials, prototypes, etc.

If a paper does not show its relevance to T-CE, it will be administratively rejected without technical review-there is no point reviewing a paper if it is out of scope. If the paper does have merit but needs extra work to prove that it is beneficial to $\mathrm{CE}$, then the paper will be administratively rejected with an invite to resubmit for technical review. T-CE is very supportive and does what it can to help authors create excellent papers for the CE community.

\section{ADVANCING CE TECHNOLOGY}

Clearly, there are gray areas where the scope of two or more journals intersect. It is often at this intersection where interesting research can begin and create new CE benefits and products. In this case, authors still need to present new CE advancement rather than just enhancement in a different scope. As an example, consider a new broadcasting system - a paper that discusses the broadcasting system alone cannot be accepted in T-CE, but a paper that discusses the broadcasting system and presents new ideas on how this broadcasting technology can be deployed in CE could well be of interest because the scope of the new ideas are focused on the CE device. Papers on multimedia streaming can be an interesting topic for T-CE, but if the paper only demonstrates improvements to the streaming service provider rather than the CE device, then it has not enhanced CE technology. Clearly, for this multimedia example, the authors should demonstrate benefits for both the content provider and the CE device or service.

Obviously, T-CE papers need to advance the state of the art for CE. This means that the paper must present and demonstrate new technology or services suitable for mass market CE. Classically, authors achieve this by comparing their work to previously published work. However, if the paper only considers (cites and references) papers from non-CE journals and compares their work to papers in non-CE journals, then where is the evidence that this new technology is relevant to CE? Where a technology is very new to $C E$, then it could be envisaged that no past $C E$ research could be relevant, but in general, $C E$ papers should place themselves in the research field of $C E$ and compare their results with past $C E$ research. It is not a requirement to cite past T-CE papers, but T-CE does advise authors to demonstrate 
that their work is a clear advancement for the CE field by comparing their work to past CE research and perhaps other research too.

Often, papers are submitted to T-CE where authors have simulated their work using a standard PC, perhaps stating which CPU was used and whether it was a dual or quad core and the amount of PC memory. However, with CE in mind, CE devices tend not to use such microprocessors, and if the work is offline simulation, then surely the type of microprocessor is irrelevant. Stating the number of cores is irrelevant if there are no details of using more than one core. The amount of PC memory is largely irrelevant to the CE application, which will be much more constrained, and the simulation host may have other applications running anyway. Often, authors state the time their algorithm executes on a PC-but since CE devices are often constrained embedded devices, then execution time on a PC offers little evidence. Of course, comparing the execution time of two algorithms on the same PC gives some feel for complexity; this still does not demonstrate the work presented will run on typical CE devices. Therefore, authors should be careful in the selection of the evidence used to demonstrate their work is an advancement- papers have to show an advancement for the field of CE and placed in that context.

\section{INSTRUCTIONS FOR AUTHORS}

Far too many papers are submitted to T-CE where the authors have clearly not read the instructions. Perhaps the wrong template is used or there is some other basic breach of the instructions. In these cases, the paper cannot be published and is simply bounced back to the authors. Therefore, authors do need to spend time preparing the paper. It is common sense to read the instructions, but many do not do it.

A usual sticking point is commercialism. The instructions for authors are clear and state:

All evidence of commercialism is to be meticulously avoided in the title, text, footnotes, tables and figures of the paper. This includes company names, trademarks, registered logos and commercial part numbers. ..All commercial part numbers, whether the subject of the paper or not, are to be avoided in the title, text, tables and illustrations.

Authors often ask me why this is the case. T-CE is a journal for archiving new and fundamental CE ideas. If a technology is locked to a specific chip, manufacturer, operating system, etc., then it is unlikely that technology will be of significant use in the medium-to-long term because technology gets outdated so fast. Papers should focus on new fundamental technology that can be deployed as widely as possible, across any vendor using any suitable technology.

Another common issue is copyright. To publish a paper, one author must sign over publication rights (not intellectual property rights) to IEEE using the standard IEEE copyright transfer form. This means that authors must have the right to transfer rights to IEEE in the first place. An increasing number of papers are using images that have been captured from broadcast or movie playback. In most cases, these images are already under copyright, and authors have no right to transfer the copyright of these images 
in their paper to the IEEE. Often, these images have embedded logos that also breach the no commercialism rule. There can be difficult cases where images are taken from a database and ownership is still not clear. Authors are advised to avoid commercial images, but in the case where these cannot be avoided, then authors are to confirm the ownership of images, obtain permission from the copyright holder to use the images, and then clearly state "permission obtained from the copyright holder" at the end of each associated figure caption in the paper.

Perhaps the most debated issue is the use of Web references. The current T-CE instructions for authors does state:

Web sites, URLS, and other online sites are not permitted as references in these archival publications because they are subject to change. This policy includes references anywhere in the paper including the reference list, figures, footnotes, or main text.

The no Web references regulation was implemented because an archival journal should withstand the test of time, whereas a Web reference can be changed or even be removed. Of course, as more material is moved online, this regulation may have to be revised in the future.

I am constantly impressed that authors whose first language is not English manage to write technical papers in the English language. However, T-CE is a professional publication, and papers can be rejected for using poor English but are more likely asked to resubmit. Authors whose first language is not English are advised to seek help in the preparation of the paper, particularly concentrating on grammar and tense (past, current, and future, and to write the paper in the third person tense). Most universities have English departments, colleagues can proofread, or there are professional language proofreading services.

Each journal has its own particular style, even inside the IEEE. The instructions for authors define the associated styles for citations, format of references, paper length, biographies, typographical, and expected content. Do follow the template to the letter, that is what it is there for.

\section{ACCEPTANCES/REJECTIONS}

The accept/reject e-mails are sent out in the third to fourth week of the publication issue. About $5 \%$ of e-mails get bounced back by the remote server as invalid or unknown DNS. Authors should use a reliable e-mail service to make sure they get the notices. Papers can be accepted on first submission or, more likely, after resubmission(s). Papers can be rejected outright after a full technical review, and in that case, authors should not resubmit the paper to T-CE. Papers can be rejected but invited to resubmit after the corrections are done, and, in this case, another full review of the resubmitted paper is still done. Papers can be administratively rejected for not meeting the basic criteria, but if the paper has promise, then it will be administratively rejected with an invite to resubmit.

If a paper is invited to be resubmitted, then the authors do need to carefully consider all the comments in the review feedback. All comments have a constructive approach to improve the paper and should be 
implemented before resubmission. Where authors disagree with review comments, then they can discuss their concerns with the EiC, who can refer the case to the original anonymous reviewers. When resubmitting, make sure the updated paper is uploaded, not the original version.

\section{CONCLUSION}

For new T-CE authors, it is a good idea to get to know a journal before you write the paper-understand what the journal is looking for, its focus, style, and scope. Researching the journal should be taken just as seriously as the research work itself. Read the instructions carefully. Prepare the paper carefully. Clearly show your technology is in the scope of T-CE by demonstrating clear benefit to CE and comparing your results to previous CE research.

\section{ABOUT THE AUTHOR}

R. Simon Sherratt (sherratt@ieee.org) is the editor-in-chief of IEEE Transactions on Consumer Electronics. He is a Fellow of the IEEE and a professor of Consumer Electronics at the University of Reading, UK

\section{REFERENCES}

[1] R. S. Sherratt, "Enhancing conference papers for submission to IEEE Transactions on Consumer Electronics", IEEE Consumer Electron. Mag., vol. 2, no. 4, pp. 8-9.

[2] IEEE authorship series: How to write for technical periodicals \& conferences. [Online]. Available: http://www.ieee.org/publications_standards/publications/authors/author_guide_interactive.pdf 